\title{
Screening and Fermentation of Jinggang-mycin High-yielding Strains
}

\author{
Xiaoju Zhang. Shijie Li . Jiangrong Xiao ${ }^{1}$ \\ Department of Biology and Chemistry Wuchang shouyi university. Wuhan 430060.China \\ 187333682@qq.com
}

\begin{abstract}
Key words: Jinggang-mycin; compound mutagenic; protoplast; resistance screening Abstract: Using KN-16 as original strains, adopting compound mutagenic of ultraviolet irradiation, ${ }^{60} \mathrm{Co} \gamma$-ray irradiation, protoplast ultraviolet irradiation, measuring the content of Jinggang-mycin A by gas chromatography. The mutagenesis condition was determined by lethality testing, flask fermentation, resistance screening of Jinggang-mycin A. When ultraviolet irradiation time at 9s, ${ }^{60} \mathrm{Co} \gamma$-ray dose at $300 \mathrm{~Gy}$, we got strains of $\mathrm{K}-108$, prepared the protoplast, ultraviolet irradiation time at $15 \mathrm{~s}$, and screening of tolerated maximum Jinggang-mycin A concentration of $5.5 \mathrm{~g} / 100 \mathrm{~mL}$, We got stable high-yield strains of 2-27-1, its Jinggang-mycin A concentration in biomass was $4.48 \mathrm{~g} / 100 \mathrm{~mL}$. Compared with the original strains, the production increased $134.6 \%$. For the mutant strains in this study, the colony morphology is irregular, yellow, light-colored, and no obvious intermediate water absorbent, the growth of colonies in the resistance plate is small, this is different to traditional view.
\end{abstract}

\section{Introduction}

Jinggang-mycin is an agricultural antibiotic, which can effectively prevent and control sheath blight of rice. It has low cost, high efficiency and safety, has no resistance after being used for more than 30 years. It can be used as a precursor of diabetes medicines ${ }^{[1]}$, and has good economic benefit and social benefit in development and utilization. Jinggang-mycin producing bacteria were identified as Streptomyces hygroscopic var. Jing gang is yen ${ }^{[2]}$, the product is mainly composed of a, b, c, d, e, $\mathrm{f}$ and other components ${ }^{[3-4]}$, and then found that $\mathrm{g}$ and $\mathrm{h}^{[5]}$ components, including jinggang-mycin a to rice sheath blight activity is the strongest ${ }^{[6]}$, generally in jinggang-mycin a content to express the quality and specification of the product. At present, the fermentation titer of jinggang-mycin producing is low, and the selection of high-yielding strains is one of the main objectives of jinggang-mycin research. Entrusted by Wuhan Ko-no biotechnology co.,ltd, in order to obtain higher fermentation yield and improve the economic benefit of jinggang-mycin, we studied jinggang-mycin producing strain $\mathrm{KN}-16$, which was used by the company at present,

\section{Materials and methods}

\section{Materials}

Strain: original strain: KN - 16, provided by Wuhan Kono biotechnology co., ltd.

Medium: slant and plate medium: glucose $1 \mathrm{~g} / 100 \mathrm{~mL}, \mathrm{~L}-$ aspartic acid $0.05 \mathrm{~g} / 100 \mathrm{~mL}$, dipotassium hydrogen phosphate $0.05 \mathrm{~g} / 100 \mathrm{~mL}$, agar $1.6 \mathrm{~g} / 100 \mathrm{~mL}$, adjust $\mathrm{pH} 7.2$ before sterilization; Fermentation medium: rice flour, peanut meal, dipotassium hydrogen phosphate, etc., pH 7.2; Mycelium medium [ ${ }^{\text {7] }}$ : glucose $1.0 \mathrm{~g} / 100 \mathrm{~mL}$, yeast extract $0.4 \mathrm{~g} / 100 \mathrm{Ml}$, peptone $0.4 \mathrm{~g} /$ $100 \mathrm{~mL}, \mathrm{MgSO}_{4} \cdot 7 \mathrm{H}_{2} \mathrm{O} 0.05 \mathrm{~g} / 100 \mathrm{~mL}, \mathrm{KH}_{2} \mathrm{PO}_{4} 0.2 \mathrm{~g} / 100 \mathrm{~mL}, \mathrm{~K}_{2} \mathrm{HPO}_{4} 0.4 \mathrm{~g} / 100 \mathrm{~mL}$,glycine $0.7 \mathrm{~g} /$ $100 \mathrm{~mL}$; Regeneration medium ${ }^{[7]}$ : sucrose $20.5 \mathrm{~g} / 100 \mathrm{~mL}, \mathrm{~K}_{2} \mathrm{SO}_{4} 0.025 \mathrm{~g} / 100 \mathrm{~mL}$, casein amino acid $0.01 \mathrm{~g} / 100 \mathrm{~mL}, \mathrm{~L}$ - proline $0.01 \mathrm{~g} / 100 \mathrm{~mL}$, trace element solution $0.2 \mathrm{~g} / 100 \mathrm{~mL}$, agar $2.5 \mathrm{~g} / 100$ $\mathrm{mL}, \mathrm{KH}_{2} \mathrm{PO}_{4} 0.005 \mathrm{~g} / 100 \mathrm{~mL}, \mathrm{CaCl}_{2} \cdot 2 \mathrm{H}_{2} \mathrm{O} 0.368 \mathrm{~g} / 100 \mathrm{~mL}$ ( separate sterilization )

\footnotetext{
${ }^{1}$ corresponding author
} 


\section{Main instruments}

Thermostatic shaker: Shanghai Zhicheng analytical instrument co., ltd; Constant temperature incubator: Shanghai Zhicheng analytical instrument co., ltd; Biomicroscopy: Olympus, Japan; Hplc analyzer: agilent techonologies, Tokyo; Desktop high-speed centrifuge: Changsha ordinary instrument co., ltd

\section{Culture conditions}

Slant strain culture: temperature is $37^{\circ} \mathrm{C}$, time is $3-5 \mathrm{~d}$;

Plate strain culture: temperature is $37^{\circ} \mathrm{C}$, time is $5 \mathrm{~d}$;

Shaking flask fermentation conditions: scraping spores on a ring of inclined surfaces into 500 mLtriangular flask ( with baffle plate ) containing $60 \mathrm{~mL}$ of liquid, at $37^{\circ} \mathrm{C}$ and $110 \mathrm{r} / \mathrm{min}$ in a rotary shaking table for $110 \mathrm{~h}$.

\section{Test methods}

Flow: original strain $\rightarrow$ ultraviolet mutagenesis $\rightarrow{ }^{60} \mathrm{Co} \gamma$-ray mutagenesis $\rightarrow$ resistance screening of high yield strain $\rightarrow$ protoplast mutagenesis $\rightarrow$ resistance screening of high yield strain $\rightarrow$ stability test $\rightarrow$ jinggang-mycin high yield strain

Ultraviolet mutagenesis: take $5 \mathrm{~mL}$ of spore suspension, put it in $\Phi 90 \mathrm{~mm}$ sterile plate, open the plate cover, under the condition of magnetic stirring with ultraviolet lamp ( wavelength $253.7 \mathrm{~nm}$, power $20 \mathrm{w}$, irradiation distance $30 \mathrm{~cm}$ ), take $0.1 \mathrm{~mL}$ of different irradiation time treatment liquid diluted coating plate, culture for 5 days at $37^{\circ} \mathrm{C}$ in dark, to colony growth, live bacteria count. Based on the number of viable bacteria in the suspension without mutagenesis, the lethal rate curve was drawn with irradiation time as abscissa and lethal rate as ordinate. After the spore suspension was irradiated for a certain period of time, $0.1 \mathrm{~mL}$ of mutagenic treatment solution was diluted and coated on the plate, and cultured for 5 days at $37^{\circ} \mathrm{C}$ from light.

${ }^{60} \mathrm{Co} \gamma$-ray mutagenesis: $10 \mathrm{~mL}$ of spore suspension of uv - treated strain was directly treated with $\gamma$-ray at doses of $100 \mathrm{gy}, 300 \mathrm{gy}, 500 \mathrm{gy}$ and $700 \mathrm{gy}$, respectively. $0.1 \mathrm{~mL}$ of appropriate diluent was coated on a flat plate, cultured at $37^{\circ} \mathrm{C}$ for 5 days, the viable bacteria in each dose group were counted, the lethal rate of ${ }^{60} \mathrm{Co} \gamma$-ray mutagenesis was calculated, and the lethal rate curve was drawn. The spore suspension was irradiated at a certain dose, and $0.1 \mathrm{~mL}$ of mutagenic treatment solution was diluted and coated on the plate, and cultured at $37{ }^{\circ} \mathrm{C}$ for 5 days from light.

Primary screening and re - screening: select the single colony on the plate with fast sporulation and central water absorption type ${ }^{[8]}$ to connect with the inclined plane of test tube, and culture at $37^{\circ} \mathrm{C}$ for 3 days. The strain with fast sporulation, thick coating, no white spots on the surface and water absorption is selected for shake flask fermentation. At $37^{\circ} \mathrm{C}, 110 \mathrm{r} / \mathrm{min}$ and fermentation $110 \mathrm{~h}$, the validamycin a component content in the fermentation broth was determined, and the strains with high Jinggang yield were screened out.

Ultraviolet mutagenesis of protoplasts: $5 \mathrm{~mL}$ of prepared jinggang-mycin tolerant strain protoplasts are taken to a sterile plate with the diameter of $9 \mathrm{~cm}$, and irradiated with ultraviolet rays ( wavelength $253.7 \mathrm{~nm}$, power $20 \mathrm{w}$, irradiation distance $30 \mathrm{~cm}$ ) under the drive of a magnetic stirrer. $0.1 \mathrm{~mL}$ of protoplast liquid at different irradiation times was then applied to the regeneration medium. Culturing at $37^{\circ} \mathrm{C}$ for 5 days in the dark, counting viable bacteria after the colony grows out, taking the number of viable protoplasts which are not mutagenized as a reference, taking irradiation time as an abscissa and mortality rate as an ordinate to draw a lethal curve, and determining the ultraviolet mutagenesis time of the protoplasts.

Selection of validamycin a resistant mutant: dilute that mutagenized spore suspension and the mutagenize protoplast, respectively coating the diluted spore suspension and the mutagenized protoplast on a validamycin a culture medium plate contain different tolerance concentrations, culturing at $37^{\circ} \mathrm{Cfor} 7$ days, and growing colonies at high concentration to obtain the validamycin a resistant strain.

Detection of validamycin a component content: adopting high performance liquid chromatography: taking off the shake flask after fermentation, weighing, supplementing water with tap water to the original weight before fermentation. Scraping thallus on triangular bottle wall with sterilized bamboo 
stick, stirring, and filtering. The filtrate was diluted appropriately, filtered by $0.45 \mu \mathrm{m}$ microfiltration membrane and determined by HPLC to obtain validamycin a component content in the fermentation broth.

\section{Results and discussion}

\section{Lethal curve of UV mutagenesis}

The lethal rate curves of strain KN - 16 and protoplast K108 - 1 after UV treatment are shown in fig. 1. the lethal rate of spores increases with the irradiation time. the spores of strain $\mathrm{KN}-16$ are sensitive to UV irradiation, and the lethal rate is $81.5 \%$ after UV treatment for 9s. The death rate of protoplast K108 - 1 increased with the irradiation time, and reached $81 \%$ at $15 \mathrm{~s}$. With the increase of irradiation time, the positive mutation rate ( defined by the increase of validamycin a production as positive mutation ) gradually decreased, and the death rate of large negative mutation was correspondingly high. Therefore, the UV irradiation time was $9 \mathrm{~s}$ and $15 \mathrm{~s}$ respectively.

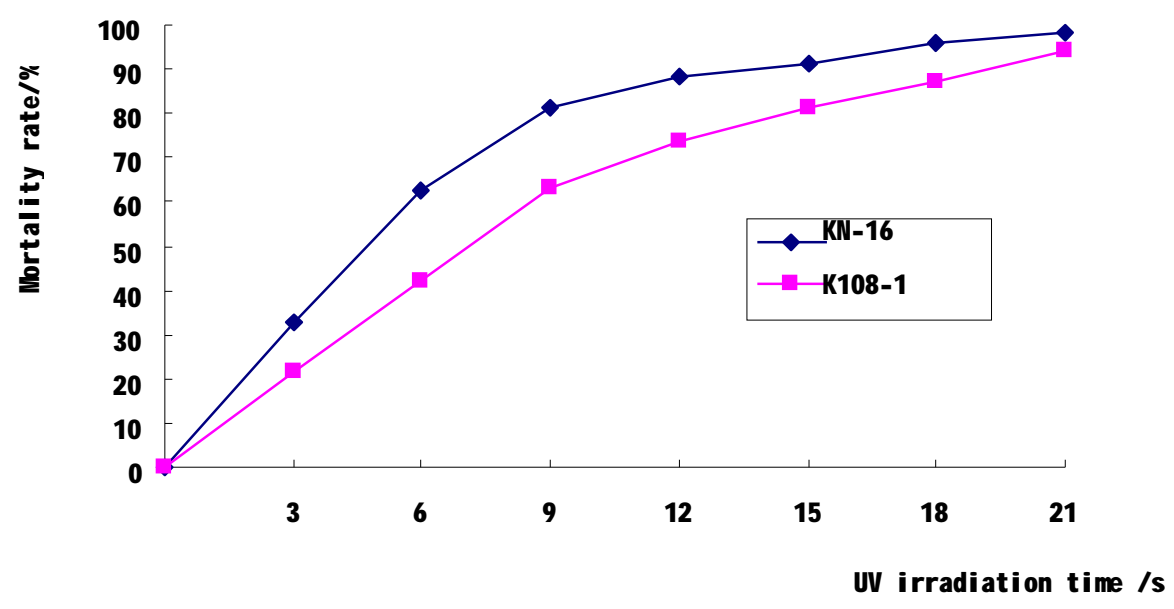

Fig.1 Mortality curve of KN-16、K108-1 treated by UV

\section{Mutagenesis of u17 spores by ${ }^{60} \mathrm{Co}-\gamma$-rays}

The lethal rate and mutation rate of $\mathrm{U} 17$ spores at different amounts of ${ }^{60} \mathrm{Co}-\gamma$ ray mutagens are shown in table 1.

Table. 1 Effect of ${ }^{60} \mathrm{Co} \gamma$ irradiation on U17

\begin{tabular}{|c|c|c|c|c|}
\hline $\begin{array}{cc}\text { Dose } & \text { of } \\
60 \mathrm{Co} \gamma / \mathrm{Gy}\end{array}$ & 100 & 300 & 500 & 700 \\
\hline $\begin{array}{l}\text { Mortality } \\
\text { rate } / \%\end{array}$ & 71.2 & 87.8 & 95.1 & 98.0 \\
\hline $\begin{array}{l}\text { Positive } \\
\text { mutation rate } 1 \%\end{array}$ & 11.7 & 13.3 & 0 & 0 \\
\hline
\end{tabular}

When the irradiation dose was $300 \mathrm{gy}$, the lethal rate of u17 spores was $87.8 \%$ and the positive mutation rate of yield was $13.3 \%$, indicating that $\mathrm{U} 17$ spores were sensitive to ${ }^{60} \mathrm{Co} \gamma$ rays. The dose of 300 gy was chosen as the optimal dose of mutagen. The spore suspension prepared by U17 was mutagenized by 300 gy ${ }^{60} \mathrm{Co}-\gamma$ ray, and then the positive mutant $\mathrm{K}-108$ was screened by shake flask fermentation as the starting strain for the next round of mutagenesis.

\section{Protoplast mutagenesis screening}

Protoplast morphology: using K - 108 as the starting strain to culture mycelium, lysozyme enzymolysis mycelium after $1 \mathrm{~h}$ centrifugation, filtration, get more complete spherical protoplast, micrograph as shown in figure 2 . 

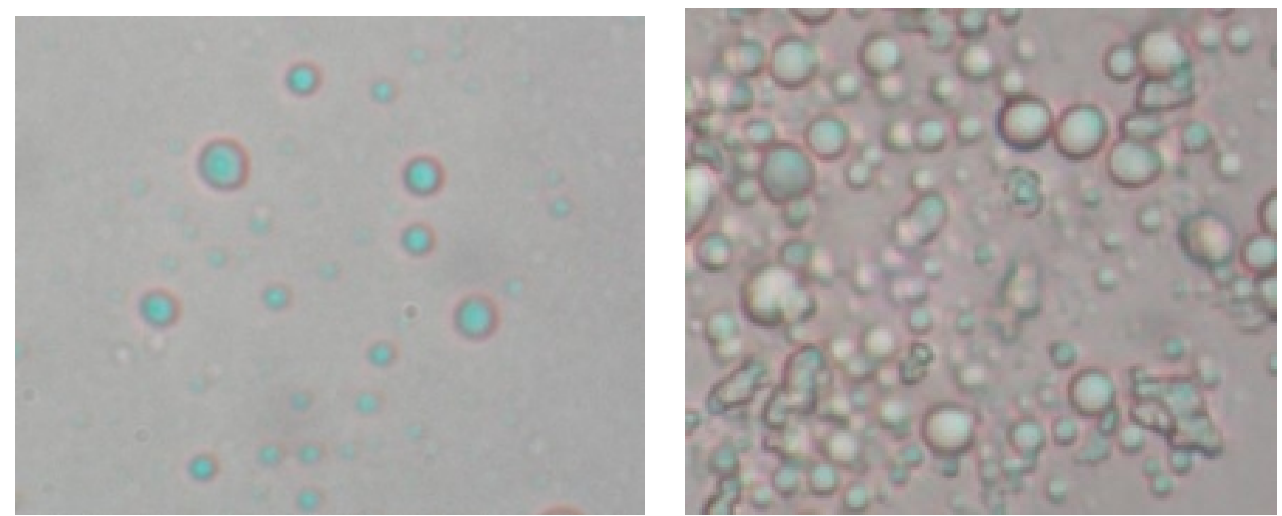

Fig.2 Protoplast morphology

UV mutagenesis of protoplasts: the prepared protoplast K108 - 1 was irradiated by UV for different time. the relationship between irradiation time and lethal rate is shown in fig. $1.15 \mathrm{~s}$ with lethal rate of $81 \%$ was selected as irradiation mutagenesis time of protoplasts.

\section{Screening of jinggangmycin a resistant mutant}

In this study, the high-yield strain $2-27-1$ was obtained by screening jinggang-mycin a resistant mutants of $\mathrm{Co}-40, \mathrm{~K} 108, \mathrm{~K} 108$ - 1 and K108 - 1 protoplasts respectively. The fermentation yields of each strain validamycin a and the description of the relevant colony morphology are shown in table 2.

Table 2 Resistance screening of Jinggang-mycin A

\begin{tabular}{|c|c|c|c|c|c|c|c|}
\hline $\begin{array}{l}\text { Jinggang-mycin A } \\
\text { content } \\
(\mathrm{g} / 100 \mathrm{~mL})\end{array}$ & 2 & & 3 & 3.4 & 3.6 & 4.1 & 5.5 \\
\hline strains & Co-40 & Co-40 & K108 & K108-1 & $\begin{array}{c}\text { K108- } \\
1\end{array}$ & $2-27$ & $2-27-1$ \\
\hline growth & + & - & + & ++ & - & ++ & + \\
\hline $\begin{array}{c}\text { Colony } \\
\text { characteristics }\end{array}$ & $\begin{array}{l}\text { white; } \\
\text { spores } \\
\text { grayed }\end{array}$ & - & $\begin{array}{l}\text { Yellow, light } \\
\text { yellow; } \\
\text { spore } \\
\text { produced }\end{array}$ & $\begin{array}{l}\text { White, } \\
\text { small } \\
\text { colony, } \\
\text { grayed after } \\
7 \text { days }\end{array}$ & - & $\begin{array}{l}\text { Round; white } \\
\text { spores; no } \\
\text { water } \\
\text { absorption }\end{array}$ & $\begin{array}{c}\text { Small; } \\
\text { irregular; no } \\
\text { water } \\
\text { absorption }\end{array}$ \\
\hline $\begin{array}{c}\text { Jinggang-mycin } \\
\text { production } \\
(\mathrm{g} / 100 \mathrm{~mL})\end{array}$ & 2.10 & - & 2.18 & 1.96 & - & 2.30 & 2.34 \\
\hline
\end{tabular}

As can be seen from table 2, the high-yield strain $\mathrm{K}-108$ was obtained by colony and resistance screening of Co - 40 spores, with an average yield of $2.18 \%$. the protoplast K108 - 1 was prepared, and the maximum concentration of validamycin a tolerance was $3.6 \mathrm{~g} / 100 \mathrm{~mL}$. after UV mutagenesis, the mutated protoplast was screened to obtain K108 - 1. Through the screening of validamycin a plate with the concentration of $5.5 \mathrm{~g} / 100 \mathrm{~mL}$, a batch of high yield strains were obtained, of which 2 - 27 1 yield reached $2.34 \mathrm{~g} / 100 \mathrm{ml}$.

\section{Conclusions}

In this study, the mutagenesis and breeding of Jinggang-mycin producing bacteria were studied systematically, and the following conclusions were obtained.

1 ) Mutagenesis system: ultraviolet mutagenesis $\rightarrow 60$ Co $\gamma$ - ray mutagenesis $\rightarrow$ screening of mutant resistant to own products $\rightarrow$ protoplast mutagenesis $\rightarrow$ screening of jinggangmycin a resistance step by step $\rightarrow$ mutant 2 - 27 - 1 with high yield and stable genetic performance. The results 
showed that Jinggang-mycin producing strain was sensitive to UV and co- irradiation, and the protoplast mutagenesis effect was significant.

2 ) The resistance screening method of Jinggangmycin A, a self-resistant product, was used twice in this study, and the high-yield mutant strains K108 and 2 - 27 - 1 were screened effectively. There are no related records of Jinggang-mycin a resistance screening in the literature, which belongs to the second innovation point of this study.

3 )Traditionally, the yield of Jinggang-mycin was higher in the colonies with good sporulation, dark brown color and good hydroscopicity. The high-yield mutants were different from the traditional ones in the aspects of irregular colony morphology, yellow, light color, no obvious phenomenon of intermediate water absorption, and small colony growth on resistant plates, which was the keyconclusion of this study and laid a foundation for the future screening of strains.

4 ) This study can be improved from the following aspects: we can consider using molecular biology to transform its genetic material and enhance its ability of producing protein; The amplification conditions of the high-yield strain obtained by mutagenesis were further optimized and controlled, especially the dissolved oxygen demand for the growth of the strain in the early stage was further studied so as to greatly shorten the fermentation period; In order to explore the high-yield performance of the induced high-yield strains, the feeding technology of the induced high-yield strains was studied.

\section{References}

[1] Hui Xu \& Jongtae Yang \& Linquan Bai \& Zixin Deng \& Taifo Mahmud Genetically engineered production of 1,1'-bis-valienamine and validienamycin in Streptomyces hygroscopicus and their conversion to valienamine. Appl Microbiol Biotechnol (2009) 81:895-902)

[2] Shanghai pesticide research institute of agricultural resistance group. jinggangmycin - producing bacteria identification [J]. microbiology, 1975,15 ( 2 ): 110 - 113.

[3] Isawa T,Kameda Y,Asai M.Studies on validaymcins,new antibiotics.IV.Isolation and characterization of validaymcins A and B[J]. Antibiotics, 1971,24(2):119-123.

[4] G Horh S,Kameda Y, Kawahara K. Studies on validamycins, new antibiotics. Isolation and characterization of validamycins C, D, E, and F[J]. Antibiotics, 1972,24: 48-53.

[5] Asano N, Kameda Y, Matsui K,et al. Validamycin H, a new pseudo-tetrasaccharide antibiotic[J]. Antibiotics, 1990, 43(8):1039-1041.

[6] Asano N, Yamaguchi T, Kameda Y,et al. Effet of validamcins on glycohydrolases of Rhizoctonia

[7] Zou Jian. screening of jinggangmycin - producing strains by protoplast mutagenesis [J ]. modern pesticides, 2003,2 ( 2 ): $27-28$.

[8] Zou Jian. breeding of jinggangmycin - producing strain [J ]. world pesticide, 2003,25 ( 6 ): 24 25.

[9] Yu juntang, Tang xiaoxuan editor. biotechnology [ M ]. Shanghai: east China university of chemical technology press, 1991: 155 - 156. 